\title{
Large Lepton Mixings Induced by Sterile Neutrino
}

\author{
K.R.S. Balaji ${ }^{1,2}$, A. Pérez-Lorenzana ${ }^{1,3}$ and A. Yu. Smirnov ${ }^{1,4}$ \\ 1 The Abdus Salam International Centre for Theoretical Physics, I-34100, Trieste, Italy \\ 2 The Institute of Mathematical Sciences, Taramani, Chennai 600 113, India \\ ${ }^{3}$ Departamento de Física, Centro de Investigación y de Estudios Avanzados del I.P.N. \\ Apdo. Post. 14-740, 07000, México, D.F., México \\ ${ }^{4}$ Institute for Nuclear Research of Russian Academy of Sciences, Moscow 117312, Russia
}

(March, 2000)

\begin{abstract}
We assume that the mass matrix of active neutrinos has hierarchical form with small mixings, similar to quark mass matrix. We show that the large mixings between $\nu_{\mu}$ and $\nu_{\tau}$ as well as between $\nu_{e}$ and certain combination of $\nu_{\mu}$ and $\nu_{\tau}$ required by the present data can appear due to the presence of a sterile neutrino state. Two realizations of this possibility are considered: (i) Large flavor mixing appears as a result of sterile neutrino "decoupling" $\left(m_{s s} \gg m_{a}\right)$, so that the active-sterile mixings are negligible. (ii) Sterile neutrino has a mass of $O(1) \mathrm{eV}$ and its mixing with active neutrinos can be observable. In the second case, the $(3+1)$ scheme of neutrino mass, which also accommodates the LSND result, can be reproduced, provided the hierarchy of the mass matrix of active neutrinos is not strong (the ratio of largest to smallest elements is about two orders of magnitude). The enhancement of lepton mixing via coupling with sterile neutrino can be realized in Grand Unified theories with the see-saw mechanism of neutrino mass generation.
\end{abstract}

PACS: 14.60.Pq; 14.60.St

\section{INTRODUCTION}

With high confidence levels, one can conclude that the atmospheric neutrino data [1] imply large or even maximal mixing of the muon neutrino. Recent detailed studies indicate that $\nu_{\mu}$ strongly mixes with $\nu_{\tau}$ while mixing with the sterile neutrino $\nu_{s}$ is disfavoured at the $3 \sigma$ level [2]. The best global fit of the solar neutrino data is given by the LMA (large mixing angle) solution, although small mixing angle solution (SMA) is not ruled out [3]. This striking difference of large lepton mixing and a small quark mixing raises important questions about the origins of neutrino masses, quark-lepton symmetry and Grand Unification.

There has been a lot of efforts to explain the appearance of large lepton mixing and small quark mixing in an unique framework. Within the context of see-saw mechanism of neutrino mass generation [ [4, which realizes quark-lepton symmetry most closely, large lepton mixing can appear due to large mixing in the charge lepton mass matrix [0] or in Dirac 
mass matrix of neutrinos [6,7]. It can also appear from large mixing or very strong hierarchy in the Majorana mass matrix of the right handed neutrinos 8]. Large lepton mixing can be obtained by radiative corrections due to renormalization group effects in schemes with quasi-degenerate neutrinos [9]. A number of possibilities exist, where the neutrino masses and the masses of the charged fermions have different origins, e.g., small neutrino masses appear due to loop effects [10].

Notice that, the mechanisms suggested so far imply an explicit introduction of large mixing in one of the sectors of the theory, or a very strong mass hierarchy or a strong mass degeneracy or qualitatively new mechanism of neutrino mass generation [11].

Another possible difference between the quark and lepton sectors is that more than three light neutrinos may be involved in lepton mixing. Introduction of an additional sterile neutrino state is motivated by simultaneous explanation of the solar, atmospheric and LSND data 12 in terms of neutrino oscillations [13.

In this paper we address the question: can these two issues, large mixing of the active neutrinos (flavor mixing) and an existence of sterile neutrino be related? We suggest a mechanism of enhancement of the flavor mixing, which is related to the existence of a sterile neutrino.

One remark is in order. It seems there is no simple relations between masses and mixing both in quark and in lepton sectors. This may be an indication of complex origin of the masses when two or more different mechanisms give comparable contributions.

The paper is organized as follows. In the next section we formulate and discuss our context. In sect. III, we describe a mechanism of enhancement of the mixing via decoupling of sterile neutrino. In Sect. IV we consider the enhancement in the non-decoupling limit and show how the LSND result can be accommodated. In sect. V we argue that the suggested mechanism can be consistent with the see-saw mechanism and Grand Unification.

\section{FLAVOR MATRIX AND $\nu_{S}$-MIXING}

We assume that the sterile neutrino, $\nu_{s}$, exists and mixes with the active neutrinos $\nu_{\alpha}$, $(\alpha=e, \mu, \tau)$. In the basis $\left(\nu_{e}, \nu_{\mu}, \nu_{\tau}, \nu_{s}\right)$ the mass matrix can be written as

$$
\hat{M}=\left(\begin{array}{cc}
\hat{m}_{f} & \vec{\epsilon} m_{s s} \\
\vec{\epsilon}^{\dagger} m_{s s} & m_{s s}
\end{array}\right),
$$

where $\hat{m}_{f}$ is the $3 \times 3$ mass matrix of the active neutrinos which we will call the flavor matrix, $m_{s s}$ is the Majorana mass term for the sterile neutrino and the vector $\vec{\epsilon}=\left(\epsilon_{e}, \epsilon_{\mu}, \epsilon_{\tau}\right)$ parametrizes the active-sterile mixing masses in units of $m_{s s}$. The parameters $\epsilon_{\alpha}$ determine the mixing between the active and sterile neutrinos.

We assume that matrix $\hat{m}_{f}$ has a hierarchical structure with small mixings, similar to the quark mass matrix. Such a matrix is usually generated by the see-saw mechanism in the context of Grand Unified theories or in general, in the theories with quark-lepton symmetry [14]. Indeed, in the GUT the Dirac neutrino mass matrix can be similar to the up-quark mass matrix and if there are no strong mixings or hierarchy in the Majorana mass matrix of the right handed neutrinos, $\hat{M}_{R}$, the see-saw mechanism, $\hat{m}_{f} \approx-\hat{m}_{D} \hat{M}_{R}^{-1} \hat{m}_{D}^{\dagger}$, can produce $\hat{m}_{f}$, which has a structure similar to $\hat{m}_{D}$ [11]. Obviously, $\hat{m}_{f} \propto \hat{m}_{D}$, if $\hat{M}_{R} \propto \hat{m}_{D}$. Certain 
realistic mass matrices $\hat{m}_{D}$ satisfy the condition $m_{D} \cdot m_{D}^{\dagger} \propto m_{D}$, so that original structure of $\hat{m}_{D}$ is reproduced by the product. In this case $\hat{m}_{f} \propto \hat{m}_{D}$, if $\hat{M}_{R} \propto \hat{I}$, where $\hat{I}$ is the unit matrix (that is, the matrix is flavor blind). In a number of cases the see-saw mechanism leads to milder hierarchy of $\hat{m}_{f}$ (factor 2 - 3 enhancement of small elements). However, only in extreme cases near maximal mixing appears [8,15].

In what follows, we will show that the mixing of the active neutrinos with the sterile state can produce large mixings of the active neutrinos. We will consider two possibilities (two limits) which have different phenomenological consequences: (i) the decoupling limit of the sterile neutrino: when $m_{s s} \gg m_{\tau \tau}$ and $\nu_{\alpha}-\nu_{s}$ mixing is very small $(\epsilon \ll 1)$; (ii) non-decoupling limit when $m_{s s} \gtrsim m_{\tau \tau}$ and $\epsilon_{\alpha}$ are not too small, so that $\nu_{\alpha}-\nu_{s}$ mixing can be observable.

\section{LARGE MIXING VIA DECOUPLING}

Let us assume that $\epsilon_{\alpha} \ll 1$ and $m_{s s} \gg m_{\alpha \beta}$, where $m_{\alpha \beta}$ are the elements of the flavor matrix $\hat{m}_{f}$. After decoupling of $\nu_{s}$ which is equivalent to the block diagonalization of the matrix (目) we get the flavor matrix $m_{f}^{\prime}$ with elements

$$
m_{\alpha \beta}^{\prime} \cong m_{\alpha \beta}-\epsilon_{\alpha} \epsilon_{\beta} m_{s s}
$$

where the second term, $\epsilon_{\alpha} \epsilon_{\beta} m_{s s} \equiv \Delta m_{\alpha \beta}$, is the contribution from the $\nu_{s}$-mixing. We define the decoupling limit in such a way that corrections to (2) being of the order $O\left(\epsilon^{3} m_{s s}\right)$, are negligible.

We require that matrix $\hat{m}_{f}^{\prime}$ leads to oscillation parameters which can explain the atmospheric and the solar neutrino data. That is, the eigenvalues, $m_{i}(i=1,2,3)$ and the mixing angles should satisfy the following condition: $m_{3}^{2}-m_{2}^{2}=\Delta m_{A}^{2} \sim(2-6) \cdot 10^{-3} \mathrm{eV}^{2}$, $\sin ^{2} 2 \theta_{\mu \tau}=\sin ^{2} 2 \theta_{A} \gtrsim 0.88, m_{2}^{2}-m_{1}^{2} \equiv \Delta m_{\odot}^{2}$ and $\sin ^{2} 2 \theta_{e x}=\sin ^{2} 2 \theta_{\odot}$. Here $x$ refers to some combination of $\nu_{\mu}$ and $\nu_{\tau}$ and $\Delta m_{\odot}^{2}, \theta_{\odot}$ are the solar oscillation parameters from one of the region of solutions for the solar neutrino problem.

In the case of mass hierarchy: $m_{3} \approx \sqrt{\Delta m_{A}^{2}} \gg m_{2} \sim \sqrt{\Delta m_{\odot}^{2}}$, the requirement of the near to maximal mixing of $\nu_{\mu}$ and $\nu_{\tau}$ reduces to the following relation between the matrix elements:

$$
m_{\mu \mu}^{\prime} \approx m_{\mu \tau}^{\prime} \approx m_{\tau \tau}^{\prime} \approx \frac{1}{2} \sqrt{\Delta m_{A}^{2}} \equiv m_{A}
$$

where we will call $m_{A}$ the atmospheric mass scale.

Diagonalizing the $\nu_{\mu}-\nu_{\tau}$ submatrix of the mass matrix $m_{f}^{\prime}$ (the so called dominant block), we get the off-diagonal elements

$$
\begin{aligned}
& \tilde{m}_{e \mu}=\cos \theta_{A} m_{e \mu}^{\prime}-\sin \theta_{A} m_{e \tau}^{\prime} \\
& \tilde{m}_{e \tau}=\cos \theta_{A} m_{e \tau}^{\prime}+\sin \theta_{A} m_{e \mu}^{\prime}
\end{aligned}
$$

where $\theta_{A}$ is the angle responsible for oscillations of the atmospheric neutrinos, so that $\sin \theta_{A} \approx \cos \theta_{A} \approx 1 / \sqrt{2}$. The element $\tilde{m}_{e \mu}$ is immediately related to the solar neutrino oscillation parameters (under the assumption that $m_{e e}$ is very small): 


$$
\tilde{m}_{e \mu} \approx \frac{\sqrt{\Delta m_{\odot}^{2}}}{2}\left[\frac{\tan ^{2} 2 \theta_{\odot}}{\sqrt{1+\tan ^{2} 2 \theta_{\odot}}}\right]^{1 / 2} .
$$

For LMA solution, we need to have typically $\tilde{m}_{e \mu}=(2-10) \cdot 10^{-3} \mathrm{eV}$, for SMA: $\tilde{m}_{e \mu}$ $=(0.3-1.5) \cdot 10^{-4} \mathrm{eV}$, and for $\mathrm{LOW}, \tilde{m}_{e \mu}=(1-2) \cdot 10^{-4} \mathrm{eV}$.

The mass $\tilde{m}_{e \tau}$ is related to the $U_{e 3}$ element of mixing matrix by

$$
\tilde{m}_{e \tau} \approx \sqrt{\Delta m_{A}^{2}} U_{e 3}
$$

which in turn is restricted by the CHOOZ result, $U_{e 3} \lesssim 0.1$, so that $\tilde{m}_{e \tau} \lesssim(4-8) \cdot 10^{-3} \mathrm{eV}$.

Phenomenological requirements (5,6) and, especially (3), can not be satisfied for arbitrary original matrix $\hat{m}_{f}$. However, as we will show, they can be satisfied due to $\nu_{s}$-mixing effect for a wide class of rather plausible matrices $\hat{m}_{f}$ with hierarchy and small mixing. Parameters of the $\nu_{s^{-}}$mixing described by $\epsilon_{\alpha}$ depend on structure of the matrix $\hat{m}_{f}$. Here, we consider three examples which differ by scale of masses in $\hat{m}_{f}$.

\section{A. Large masses in $\hat{m}_{f}$}

Suppose that

$$
m_{\tau \tau} \gg m_{\tau \mu} \gg m_{\mu \mu} \sim m_{A}
$$

(two largest elements in $\hat{m}_{f}$ are much heavier than the atmospheric mass scale). For instance, the elements of $\hat{m}_{f}$ may have the following hierarchical structure: $m_{\tau \tau}=m_{0} \sim O(10 \mathrm{eV})$, $m_{\tau \mu} \sim m_{0} \lambda^{2}, m_{\mu \mu} \sim m_{e \tau} \sim m_{0} \lambda^{4}, m_{e \mu} \sim m_{0} \lambda^{6}, m_{e e} \sim m_{0} \lambda^{8}$, where $\lambda \sim 0.2$, is the hierarchy parameter of the order of Cabibbo angle.

The contribution from the $\nu_{s}$-mixing should lead to strong cancellation in $m_{\tau \tau}^{\prime}$ and $m_{\tau \mu}^{\prime}$. From the condition $m_{\tau \tau}^{\prime} \sim m_{\tau \mu}^{\prime} \sim m_{A}$ we get

$$
\epsilon_{\tau} \cong \sqrt{\frac{m_{\tau \tau}}{m_{s s}}}, \quad \frac{\epsilon_{\mu}}{\epsilon_{\tau}} \cong \frac{m_{\tau \mu}}{m_{\tau \tau}} \sim \lambda^{2} .
$$

Furthermore, using (8) we find for the element $m_{\mu \mu}^{\prime}$

$$
m_{\mu \mu}^{\prime}=m_{\mu \mu}-\frac{m_{\tau \mu}^{2}}{m_{\tau \tau}} \sim m_{A}
$$

and in fact, $m_{\mu \mu}$ can be much smaller than $m_{A}$. Notice that the signs of $m_{\mu \mu}^{\prime}$ and $m_{\tau \tau}^{\prime}$ should be the same, which can be realized if $\left|m_{\tau \tau}\right|<\left|\epsilon_{\tau}^{2} m_{s s}\right|$

In our example of $m_{f}(\lambda)$, we get $m_{e \mu} \ll m_{e \tau} \sim 10^{-2} \mathrm{eV}$, so that $\left|\tilde{m}_{e \mu}\right| \approx\left|\tilde{m}_{e \tau}\right| \sim 5 \cdot 10^{-3}$ $\mathrm{eV}$. These values satisfy the CHOOZ bound and lead to the LMA solution. (The expected value of $U_{e 3}$ is close to the upper bound.) Therefore, no significant contribution of the $\nu_{s}$-mixing to these elements is required, and we can set an upper bound on $\epsilon_{e}$. From the condition $\Delta m_{e \tau}=\left(\epsilon_{e} / \epsilon_{\tau}\right) m_{\tau \tau} \lesssim 10^{-2}$ eV we get

$$
\frac{\epsilon_{e}}{\epsilon_{\tau}} \lesssim 10^{-3}
$$


Actually, the $\nu_{s}$-mixing with $\epsilon_{e} / \epsilon_{\tau} \sim 10^{-3}$ can be used to suppress $U_{e 3}$ and/or to reproduce the SMA solution.

Thus, the hierarchical structure of $\nu_{s}$-mixing is required $\epsilon_{e}: \epsilon_{\mu}: \epsilon_{\tau}=\left(\lesssim 10^{-3}\right): 0.04: 1$ (in our example) which should correlate with hierarchy of the original mass matrix. This can be a consequence of certain flavor symmetry.

\section{B. Moderate masses in $\hat{m}_{f}$}

Suppose that

$$
m_{\tau \tau} \gg m_{\tau \mu} \sim m_{A}
$$

(only $m_{\tau \tau}$ is much larger that the atmospheric neutrino scale). As we will see, the contribution of the $\nu_{s}$-mixing to $m_{\mu \mu}$ is small, so that the condition (3i) can be satisfied only if

$$
m_{\mu \mu} \sim m_{A}
$$

The original flavor matrix $\hat{m}_{f}$ could have the following structure: $m_{\tau \tau}=m_{0} \sim O(1 \mathrm{eV})$, $m_{\tau \mu} \sim m_{\mu \mu} \sim m_{0} \lambda^{2}, m_{e \mu} \sim m_{e \tau} \sim m_{0} \lambda^{4}, m_{e e} \sim m_{0} \lambda^{6}$.

From the condition of cancellation in $m_{\tau \tau}^{\prime}$ we get for $\epsilon_{\tau}$ the same relation as in (8). Contribution of the $\nu_{s}$-mixing to $m_{\tau \mu}$ should not be large. Requiring $\Delta m_{\tau \mu} \lesssim m_{A}$, we find

$$
\frac{\epsilon_{\mu}}{\epsilon_{\tau}} \lesssim \frac{\sqrt{\Delta m_{A}^{2}}}{2 m_{\tau \tau}}=(2-4) \cdot 10^{-2}\left(\frac{1 \mathrm{eV}}{m_{\tau \tau}}\right) .
$$

One can check now that the correction to $m_{\mu \mu}$ is indeed small: $\Delta m_{\mu \mu} \sim\left(\epsilon_{\mu} / \epsilon_{\tau}\right)^{2} m_{\tau \tau}=$ $(0.4-1.6) \cdot 10^{-3} \ll m_{A}$.

For our example of $m_{f}(\lambda=0.2)$ we get $m_{e \mu} \sim m_{e \tau} \sim(1-2) \cdot 10^{-3} \mathrm{eV}$, so that $\left|\tilde{m}_{e \mu}\right| \approx$ $\left|\tilde{m}_{e \tau}\right| \sim(0-3) \cdot 10^{-3} \mathrm{eV}$. Therefore the CHOOZ bound is satisfied, and moreover, expected $U_{e 3}$ is much below the upper bound. Any solution for the solar neutrino problem can be reproduced depending on precise values of parameters in $\hat{m}_{f}$. Therefore, no significant contribution to these elements from the $\nu_{s}$-mixing is required. The upper bound on $\epsilon_{e}$ can be obtained from the condition, $\Delta m_{e \tau} \leq 10^{-2} \mathrm{eV}:\left(\epsilon_{e} / \epsilon_{\tau}\right) \leq 10^{-2}$. Thus, $\epsilon_{\alpha}$ are hierarchical, $\epsilon_{e}: \epsilon_{\mu}: \epsilon_{\tau}=\left(<10^{-2}\right): 0.04: 1$,although the hierarchy can be weaker than in the previous case.

Let us consider another structure of the original flavor mass matrix: $m_{e \mu} \sim m_{e \tau} \sim m_{0} \lambda^{3}$, $m_{e e} \sim m_{0} \lambda^{4}$. In this case, $m_{e \mu} \sim m_{e \tau} \sim(4-8) \cdot 10^{-3} \mathrm{eV}$, which can give $\left|U_{e 3}\right|$ at the level of the CHOOZ bound and lead to the LMA solution without any contribution from the $\nu_{s}$-mixing. For $\epsilon_{e} \sim 10^{-2} \epsilon_{\tau}$, cancellation between $m_{e \mu}^{\prime}$ and $m_{e \tau}^{\prime}$ in $\tilde{m}_{e \mu}$ allows one to reproduce also the SMA solution.

\section{Small masses in $\hat{m}_{f}$}

Suppose that 


$$
m_{\mu \mu} \ll m_{\tau \mu} \ll m_{\tau \tau} \sim m_{A}
$$

i.e., all the masses in the original flavor matrix are smaller than the atmospheric mass scale. As an example we can take $m_{\tau \tau}=m_{0} \sim 0.03 \mathrm{eV}, m_{\tau \mu} \sim m_{0} \lambda, m_{\mu \mu} \sim m_{e \tau} \sim m_{0} \lambda^{2}$, $m_{e \mu} \sim m_{0} \lambda^{3}, m_{e e} \sim m_{0} \lambda^{4}$. In this case, the entire dominant block is formed by contributions from the $\nu_{s}$-mixing and the original flavor matrix gives small corrections. In order to satisfy (3) we should take $\epsilon_{\mu} \sim \epsilon_{\tau}$. Neglecting all mass terms but $m_{\tau \tau}$ in $\hat{m}_{f}$, we have

$$
\begin{aligned}
& m_{\mu \mu}^{\prime} \approx-\epsilon_{\mu}^{2} m_{s s} ; m_{\mu \tau}^{\prime} \approx-\epsilon_{\mu} \epsilon_{\tau} m_{s s} ; \\
& m_{\tau \tau}^{\prime} \approx m_{\tau \tau}-\epsilon_{\tau}^{2} m_{s s} .
\end{aligned}
$$

Choosing $\epsilon_{\tau}^{2} m_{s s}=2 m_{\tau \tau}$, from the condition $\left|m_{\tau \tau}^{\prime}\right| \sim\left|m_{\tau \mu}^{\prime}\right| \sim m_{A}$ we get $\epsilon_{\mu} / \epsilon_{\tau}=1 / 2$.

The mass $m_{2}$ responsible for the solar neutrino conversion is determined by $m_{\tau \tau}$. The determinant of the $\nu_{\mu}-\nu_{\tau}$ submatrix equals $-\epsilon_{\mu}^{2} \epsilon_{\tau}^{2} m_{s s}^{2}-m_{\mu \mu}^{\prime} m_{\tau \tau} \approx m_{A} m_{\tau \tau} / k$, where $k=$ $O(1)$ is a free parameter. Therefore, taking into account that $m_{3} \sim 2 m_{A}$, we get

$$
m_{2} \sim \frac{m_{\tau \tau}}{2 k} \sim \frac{\sqrt{\Delta m_{A}^{2}}}{4 k} .
$$

For $k=2$ we find the mass $m_{2}=(0.5-1) \cdot 10^{-2} \mathrm{eV}$, which is well in the range of the LMA solution, furthermore $\sin ^{2} 2 \theta_{A}=0.94$.

For other elements (without $\nu_{s}$-mixing contribution) we have, $m_{e \mu} \ll m_{e \tau} \sim(1-2) \cdot 10^{-3}$ $\mathrm{eV}$, so that $\left|\tilde{m}_{e \mu}\right| \approx\left|\tilde{m}_{e \tau}\right| \sim(0.5-1) \cdot 10^{-3} \mathrm{eV}$, which is much smaller than value required by the LMA solution. However, $m_{e \mu}$ can be enhanced by the $\nu_{s}$-mixing contribution. Requiring that $\Delta m_{e \tau} \sim 6 \cdot 10^{-3} \mathrm{eV}$, we get $\epsilon_{e} / \epsilon_{\tau} \sim 1 / 10$. Thus the scheme implies a rather weak hierarchy of mixing parameters: $\epsilon_{e}: \epsilon_{\mu}: \epsilon_{\tau}=0.1: 0.5: 1$.

\section{On the scale of $m_{s s}$}

Clearly, all the contributions from the $\nu_{s}$-mixing will be unchanged if $\epsilon_{\alpha}$ decreases with increase of $m_{s s}$ as

$$
\epsilon_{\alpha}=\epsilon_{\alpha}^{0} \sqrt{\frac{m_{s s}^{0}}{m_{s s}}} .
$$

In our examples the largest $\epsilon_{\alpha}$ is $\epsilon_{\tau}=\sqrt{m_{\tau \tau} / m_{s s}}$, and the decoupling limit holds if $\epsilon_{\tau}<\left(10^{-1}-10^{-2}\right)$ depending on the type of the flavor mass matrix. Let us consider several possibilities.

For $m_{\tau \tau} \sim 1 \mathrm{eV}$, the decoupling implies that $m_{s s}$ should be at least in the kev range. The kev sterile neutrinos with even very small mixing (as small as $\epsilon \sim 10^{-2}-10^{-4}$ ) may have important consequences both in astrophysics and cosmology. They have been discussed as a possible component of the (warm) dark matter of the universe [16], in connection with pulsar kick problem [17,18], the proton loading problem for gamma bursters [19]. Such neutrinos can be produced in the conversion of active neutrinos in the supernovae and in the early Universe. To satisfy the cosmological bound these neutrinos should decay during the time 
shorter than the lifetime of the universe or one should assume that these neutrinos as well as active neutrinos were never in thermal equilibrium [20].

If $m_{s s} \sim 1 \mathrm{TeV}$, which one could expect for singlets in the supergravity models (modulinos) 21 then $\epsilon_{\tau} \sim 10^{-6}$, so that the mixing terms $\epsilon_{\tau} m_{s s}$ should be about $1 \mathrm{MeV}$. For $m_{s s} \sim 10^{13} \mathrm{GeV}$, we have $\epsilon_{\tau} \sim 10^{-11}$ and $\epsilon_{\tau} m_{s s} \sim 100 \mathrm{GeV}$, which corresponds to the electroweak scale.

\section{NON-DECOUPLING LIMIT AND LSND RESULT}

\section{A. Without LSND result}

Here we will assume that $m_{s s} \sim(1-10) \mathrm{eV}$. In the case of strong hierarchy of the mixing parameters $\epsilon_{\tau} \gg \epsilon_{\mu} \gg \epsilon_{e}$ as in examples 1) and 2) from the previous section the non-decoupling effect reduces to relatively large mixing of the $\nu_{\tau}$ and $\nu_{s}$ in the fourth mass eigenstate. So, in principle, one can observe $\nu_{\tau} \leftrightarrow \nu_{s}$ oscillations driven by $\Delta m^{2} \sim m_{s s}^{2}$ in the short base-line experiments. All other masses and mixings will be as in the decoupling limit.

Let us now discuss a possibility to realize the $(3+1)$ scheme [22] with relatively large $\epsilon_{e}$ and $\epsilon_{\mu}$ which can also accommodate the LSND result. Now block diagonalization is impossible and we need to consider whole $4 \times 4$ matrix $\hat{M}$.

First of all, it is easy to see that if the flavor matrix $\hat{m}_{f}$, has a strong hierarchy, the LSND result can not be reproduced because of the CHOOZ bound. Let us consider the matrix $\hat{m}_{f}$ with only one non-zero element, $m_{\tau \tau}$. As follows from (1), in this $m_{\tau \tau}$ dominance approximation one state

$$
\nu_{d} \equiv \cos \phi \nu_{e}-\sin \phi \nu_{\mu}
$$

where $\cos \phi=\epsilon_{\mu} / \sqrt{\epsilon_{e}^{2}+\epsilon_{\mu}^{2}}$ decouples and the orthogonal state

$$
\nu_{n} \equiv \sin \phi \nu_{e}+\cos \phi \nu_{\mu}
$$

will have the mixing element, $\sqrt{\epsilon_{e}^{2}+\epsilon_{\mu}^{2}} m_{s s}$ in the rest $3 \times 3$ matrix. To explain the atmospheric neutrino data, one needs to have large admixture of $\nu_{n}$ (which contains $\nu_{\mu}$ ) in the $\nu_{3}$ - state. Denoting by $U_{n 3}$, this admixture we get

$$
U_{\mu 3}=\cos \phi \cdot U_{n 3}, \quad U_{e 3}=\sin \phi \cdot U_{n 3} \cdot
$$

From (20), we get

$$
U_{e 3}=U_{\mu 3} \cdot \tan \phi=U_{\mu 3} \cdot \frac{\epsilon_{e}}{\epsilon_{\mu}}
$$

where the atmospheric data imply $U_{\mu 3} \gtrsim 0.5$. The LSND result, in turn, requires $\epsilon_{e} \sim$ $0.12-0.14$ and $\epsilon_{\mu} \sim 0.12-0.16$, so that from (21) we get $\left|U_{e 3}\right|^{2}>0.12$ which is certainly excluded by CHOOZ result [23]. Thus, large mixing of $\nu_{\mu}$ in $\nu_{3}$ leads simultaneously to large mixing of $\nu_{e}$ in $\nu_{3}$.

Taking $\epsilon_{e} / \epsilon_{\mu} \sim 1 / 3$ to statisfy the CHOOZ limit, we get for $\epsilon_{\mu} \leq 0.15, \sin ^{2} 2 \theta_{L S N D} \approx$ $4 \epsilon_{e}^{2} \epsilon_{\mu}^{2} \leq 2.5 \cdot 10^{-4}$, which is smaller than the experimental result [24]. This requires a departure from the $m_{\tau \tau}$ dominance scheme. 


\section{B. With LSND result}

In what follows, we show that the LSND result can be reproduced if the flavor matrix has a moderate hierarchy:

$$
M_{\alpha \beta} \sim \frac{1}{3} M_{\beta \beta}
$$

where $\alpha$ corresponds to lighter flavor than $\beta$.

The mass matrix in the flavor basis can be written as

$$
\hat{M}=\hat{U} \hat{M}^{\operatorname{diag}} \hat{U}^{\dagger}
$$

where $\hat{U}$ is the mixing matrix, and the diagonal matrix of the mass eigenvalues, $\hat{M}^{\text {diag }}$, can be parametrized as

$$
\hat{M}^{\text {diag }}=m_{1} \hat{I}+\operatorname{diag}(0, \delta, 0,0)+\operatorname{diag}(0,0, \Delta, m),
$$

so that $m_{2}=m_{1}+\delta, m_{3}=m_{1}+\Delta$, and $m_{4}=m_{1}+m$. Inserting (24) into (23) we get

$$
\hat{M}=m_{1} \hat{I}+\hat{M}^{\delta}+\hat{U} \operatorname{diag}(0,0, \Delta, m) \hat{U}^{\dagger},
$$

where $\hat{M}^{\delta} \equiv \hat{U} \operatorname{diag}(0, \delta, 0,0) \hat{U}^{\dagger}$.

Let us first assume that $m_{1} \gg \delta$, so that two light states are degenerate, $m_{1} \approx m_{2}$. Neglecting $\hat{M}^{\delta}$, we get for the elements of matrix $\hat{M}$ :

$$
M_{\alpha \beta}=m_{1} \delta_{\alpha \beta}+\Delta U_{\alpha 3} U_{\beta 3}^{\dagger}+m U_{\alpha 4} U_{\beta 4}^{\dagger} .
$$

That is, the structure of the mass matrix is determined by the mass of the degenerate pair, $m_{1}$, by mass parameters $\Delta$ and $m$ and by flavour mixing in the third and in the fourth mass eigenstates $U_{\alpha 3}, U_{\alpha 4}$. These mixing elements are, in turn, determined by phenomenology and a condition of the mass hierarchy. Indeed, the LSND result requires admixtures of the $\nu_{e}$ and $\nu_{\mu}$ in $\nu_{4}$ to be at the level of upper experimental bounds: $U_{e 4} \sim 0.10-0.14$ [25], $U_{\mu 4} \sim 0.12-0.15$ [26]. The element $U_{e 3}$ is restricted by the CHOOZ result: $U_{e 3}<0.1$ [23]; $U_{\mu 3}$ is given by the atmospheric neutrino data: $U_{\mu 3}=(0.55-0.85)$. One mixing parameter (we take $U_{\tau 3}$ ) is free, and all other parameters are determined by the unitarity condition (orthogonality of $\nu_{3}$ and $\nu_{4}$ and normalization: $\sum\left|U_{\alpha 3}\right|^{2}=\sum\left|U_{\alpha 4}\right|^{2}=1$ ).

Three lightest elements of the flavor mass matrix can then be written as

$$
\begin{aligned}
& M_{e e} \approx m_{1}+m\left|U_{e 4}\right|^{2} \approx 0.014 m+m_{1} \\
& M_{e \mu} \approx m U_{e 4} U_{\mu 4}^{\dagger} \approx 0.017 m \\
& M_{\mu \mu} \approx m_{1}+\Delta\left|U_{\mu 3}\right|^{2}+m\left|U_{\mu 4}\right|^{2}
\end{aligned}
$$

where the contributions of terms proportional to $\Delta$ ( $\Delta$-terms) to $M_{e e}$ and $M_{e \mu}$ can be safely neglected. Clearly, to achieve the hierarchy, $M_{e e} \ll M_{e \mu} \ll M_{\mu \mu}$, one needs significant cancellation in $M_{e e}$, and therefore

$$
m_{1}=-(0.01-0.02) m \sim-(0.01 \div 0.02) \mathrm{eV}
$$


Furthermore, $\Delta$ - term in (27) should give a significant contribution to $M_{\mu \mu}$ : for $\Delta=7 \cdot 10^{-2}$ $\mathrm{eV}$ and $\left|U_{\mu 3}\right|^{2} \approx 0.5$ we get $M_{\mu \mu} \sim 0.05 \mathrm{~m}$, which is about factor of three larger than $M_{e \mu}$.

For $M_{\tau \tau}$ - element, we have

$$
M_{\tau \tau} \approx m_{1}+\Delta\left|U_{\tau 3}\right|^{2}+m\left|U_{\tau 4}\right|^{2}
$$

Hierarchy between the diagonal elements, $M_{\tau \tau} \gg M_{\mu \mu}$, implies that the third term in (29) should dominate over others. This means that the admixture of $\nu_{\tau}$ in the fourth state should not be small. From the condition $M_{\tau \tau} \geq(5-10) M_{\mu \mu}$, we get

$$
\left|U_{\tau 4}\right| \geq 0.4-0.7
$$

Dominance of the terms proportional to $m$ ( $m$ - terms) in the elements $M_{\alpha \tau}$ leads to relations

$$
M_{e \tau}: M_{\mu \tau}: M_{\tau \tau}=\left|U_{e 4}\right|:\left|U_{\mu 4}\right|:\left|U_{\tau 4}\right| .
$$

As an example we may have $M_{e \tau}: M_{\mu \tau}: M_{\tau \tau}=0.20: 0.25: 1$. For values of mixing (30) we get, $M_{\tau \tau}=(0.17-0.45) \mathrm{eV}$ (if $\left.m=1 \mathrm{eV}\right)$.

We evaluate the effect of matrix $M^{\delta}$ in (25). The parameter $\delta$, splits the light degenerate mass eigenvalues and, the size of $\delta$ is determined by the solar mass splitting $\Delta m_{\odot}^{2}$. For $m_{1}=10^{-2} \mathrm{eV}$ and $\Delta m_{\odot}^{2}=(4-6) \cdot 10^{-5} \mathrm{eV}^{2}$ (which is typical for LMA solution) we get

$$
\delta \approx \frac{\Delta m_{\odot}^{2}}{2 m_{1}} \approx(2-3) \cdot 10^{-3} \mathrm{eV}
$$

For all other solutions value of $\delta$ and consequently the corrections are smaller. Given, $M_{\alpha \beta}^{\delta}=\delta \cdot U_{\alpha 2} U_{\beta 2}^{\dagger}$, the largest mass term equals $M^{\delta} \leq 0.5 \delta \sim(1-2) \cdot 10^{-3} \mathrm{eV}$. Thus, the corrections are small even for the smallest element: $M_{e e}$. For the remaining elements, the corrections are negligible and can not influence the structure of the mass matrix. These small corrections are, however, important for fixing the oscillation parameters relevant for solar neutrinos.

Let us comment on the case of strong hierarchy of the mass eigenvalues. This would correspond to the limit $m_{1} \ll \delta$ and

$$
\delta \approx \sqrt{\Delta m_{\odot}^{2}} \approx(5-10) \cdot 10^{-3} \mathrm{eV}
$$

Now the $M_{e e}$-element of the mass matrix equals

$$
M_{e e} \approx \delta\left|U_{e 2}\right|^{2}+m\left|U_{e 4}\right|^{2}
$$

and according to (33) the first term in (34) is about $(2-5) \cdot 10^{-3} \mathrm{eV}$. It can not compensate the second term that is required by the hierarchy $M_{e e} \ll M_{e \mu}$. The compensation can be achieved if $\delta \geq 2 \cdot 10^{-2} \mathrm{eV}$ which would correspond to $\Delta m_{\odot}^{2} \geq 4 \cdot 10^{-4} \mathrm{eV}^{2}$. This value is disfavored by present data, although not excluded completely.

Collecting all the information discussed above we get as an example the following mass matrix 


$$
\hat{M} \approx m\left(\begin{array}{cccc}
0.004 & 0.0168 & 0.081 & 0.14 \\
0.0168 & 0.05 & 0.13 & 0.142 \\
0.081 & 0.13 & 0.385 & 0.6 \\
0.14 & 0.142 & 0.6 & 1
\end{array}\right)
$$

For $m=1 \mathrm{eV}$ this matrix leads to $\sin ^{2} 2 \theta_{\odot}=0.92 ; \Delta m_{\odot}^{2}=4.6 \cdot 10^{-5} \mathrm{eV}^{2} ; \sin ^{2} 2 \theta_{A}=$ $0.958 ; \Delta m_{A}^{2}=3.1 \cdot 10^{-3} \mathrm{eV}^{2} ; \sin ^{2} 2 \theta_{L S N D}=1 \cdot 10^{-3}$. We also get $U_{e 3}=0.099$. To illustrate the role of the sterile neutrino couplings we find that in their absence the active part in Eq. (35) alone provides for atmospheric neutrinos: $\Delta m^{2}=0.19 \mathrm{eV}^{2}$ and $\sin ^{2} 2 \theta=0.35$, which are far beyond the required values, and for solar neutrinos: $\Delta m^{2}=10^{-4} \mathrm{eV}^{2}$ and $\sin ^{2} 2 \theta=0.55$.

Thus, the $(3+1)$ scheme can be realized if the flavor mass matrix has a moderate hierarchy (22). There are two generic consequences of the scheme: (i) the pair of the light states should be degenerate with $m_{1} \approx m_{2} \approx(0.01-0.02) \mathrm{eV}$. This leads to the effective Majorana mass of the electron neutrino

$$
m_{e e}=m_{1} \cos 2 \theta_{\odot}
$$

which can be as large as $\sim O\left(10^{-2}\right) \mathrm{eV}$ if the solar mixing is not to close to maximal one. (ii) The scheme predicts rather large mixing of $\nu_{\tau}$ and $\nu_{s}$ in the heaviest eigenstate (30), so that oscillation $\nu_{\tau} \leftrightarrow \nu_{s}$ drived by $\Delta m^{2} \sim \Delta m_{L S N D}^{2}$ should be observed.

\section{V. $\nu_{S}$-MIXING AND THE SEE-SAW MECHANISM}

Let us show that $\nu_{s}$-mixing considered in the previous sections is consistent with the see-saw mechanism for the mass generation of the active neutrinos.

There are several possibilities to couple the sterile neutrinos with the active neutrinos. The simplest one is the direct coupling through the Yukawa interaction $h_{s} \bar{\nu}_{L} \nu_{s} H$, where $H$ is the Higgs doublet. In this case, however, one needs a very tiny coupling constant, $h_{s} \approx \epsilon m_{s s} /\langle H\rangle \approx 5 \cdot 10^{-13}$, and an additional mechanism is required to explain such a small value.

Another possibility is the indirect mixing via coupling of $\nu_{s}$ with heavy right handed neutrinos $N_{R}$ [27]. Let us consider the following mass terms

$$
-\mathcal{L}=\bar{\nu}_{L} m_{D} N_{R}+N_{R}^{T} M_{R} N_{R}+\bar{\nu}_{s} \mu^{\dagger} N_{R}+m_{s} \nu_{s}^{T} \nu_{s}+\text { h.c. },
$$

where $\mu$ is the mass parameter of the $\nu_{s}$ and $N_{R}$ mixing. After decoupling of the right handed neutrinos the matrix $\hat{M}$ is produced with the folowing elements

$$
\begin{aligned}
& \hat{m}_{f}=-m_{D} M_{R}^{-1} m_{D}^{\dagger} ; \quad \epsilon_{\alpha} m_{s s}=-\left[m_{D}^{\dagger} M_{R}^{-1}\right]_{\alpha \beta} \mu_{\beta} ; \\
& m_{s s}=m_{s}-\mu^{\dagger} M_{R}^{-1} \mu
\end{aligned}
$$

The values of $\epsilon_{\alpha}$ are determined by $\mu_{\alpha}$, with a scaling factor $\langle H\rangle / M$. In order for $\epsilon_{\alpha}$ to be small enough we need $\mu$ to be at the level of the $m_{D}$ scale. Also the corrections to $m_{s s}$ should be sufficiently suppressed by the right handed mass scale. 
The matrix $\hat{M}$ formed at the scale $M_{R}$ will be renormalized by the radiative corrections. In particular, the gauge (weak) interactions will renormalize differently the flavor submatrix and the mixing mass vector:

$$
\hat{m}_{f} \rightarrow r_{g} \hat{m}_{f}, \quad \epsilon_{\alpha} m_{s s} \rightarrow \sqrt{r_{g}} \epsilon_{\alpha} m_{s s},
$$

where $r_{g} \sim(1-2)$ is the renormalization factor which is model dependent. Clearly, $m_{s s}$ is unchanged. Yukawa couplings can produce rather small renormalization effect (mainly of the mass terms $M_{a \tau}$, where $\left.a=e, \mu, \tau, s\right)$. The renormalization should be taken into account when fixing parameters at the high (probably GUT) mass scale.

In the non-decoupling limit, $m_{s s}$ should be small. Such a small mass can be explained by the see-saw mechanism in the "sterile sector". The scale can be protected by some additional $U(1)$ symmetry, which is broken at $\sim(1-100) \mathrm{TeV}$ scale [28]. In the context of the supersymmetric theories a smallness of $m_{s s}$ can be related to physics of SUSY breaking [29].

\section{DISCUSSION AND CONCLUSION}

We have shown that mixing between active neutrinos can be enhanced by the presence of sterile neutrino. In particular, large or even maximal mixing can be induced in the flavor mass matrix with original strong mass hierarchy and small mixing.

A simultaneous explanation of the atmospheric and solar neutrino data requires however certain structure of the original flavor mass matrix (or the introduction of more than one sterile neutrino). For hierarchical flavor mass matrix of general form this is impossible. Indeed, mixing with just one sterile neutrino produces three independent mass terms, whereas four oscillations parameters (two $\Delta m^{2}$ and two mixing angles) should be explained.

We have considered several cases which differ by the scale of elements in the original flavor mass matrix (larger, of the order, or smaller than the atmospheric neutrino scale). For each case we have found the relevant structure of the flavor mass matrix. These structures look rather plausible from the point of view of theories with flavor symmetries. Clearly, more freedom appears if there are additional sterile neutrinos.

Near maximal mixing appears as an interplay of the original flavor matrix and the mass matrix induced by mixing with sterile neutrino. If the origin of the mixing terms $(\epsilon)$ is unrelated to the origin of the flavor mass matrix, then near maximal mixing appears accidental. In fact, in the case of mixing via right handed neutrinos (37) the mixing terms may not be completely independent of the elements of flavor matrix: both of them are proportional to the product $m_{D} M_{R}^{-1}$. The required relations between the $\epsilon$ - terms and the flavor matrix at some high scale may be rather non-trivial in view of renormalization effects.

In our framework there is no simple symmetry which leads to maximal mixing. However it is not excluded that the required relation between $\epsilon$ terms and the flavor matrix appears in the underlying theory of flavor. An exception is the case IIIC, where both masses and mixing relevant for the atmospheric neutrino oscillations are given by the dominant contribution from the sterile to active neutrino mixings. In this case near maximal flavor mixing can appear due to certain symmetry (equality) of the $\epsilon$-elements themselves.

In this paper we have concentrated on the phenomenological aspects of the mechanism. Depending on the mass of sterile neutrino, two limits have been considered. In the decoupling 
limit, mixings of active and sterile neutrinos are very small. They are negligible for the laboratory experiments. The effect of sterile neutrinos is just reduced to correction of the mass matrix of the active neutrinos. However, the sterile neutrinos with masses in $(1-100)$ kev range and even very small mixing can have important implications for astrophysics and cosmology.

In the non-decoupling limit, large lepton mixings is accompanied by an observable activesterile neutrino mixing. Moreover, for $\hat{m}_{f}$ with moderate hierarchy the $(3+1)$ scheme of neutrino mass 22] can be realized which accommodate oscillation solutions of the atmospheric and solar neutrino problems and the LSND result. The generic prediction of such a scheme is a large $\nu_{e}-\nu_{s}$ mixing which leads to observable $\nu_{e} \leftrightarrow \nu_{s}$ oscillations.

The suggested mechanism of enhancement of mixing can reconcile large observed mixings and the hierarchical structure of the active neutrino mass matrix (having small mixings), which naturally follows from the see-saw mechanism in the context of the Grand Unified Theories.

Balaji wishes to thank the High Energy Theory group at The Abdus Salam ICTP for local support and for the hospitality at the Theoretical Physics Division, University of Helsinki, where this work was completed. 


\section{REFERENCES}

[1] Super-Kamiokande Collab., Y. Fukuda et al., Phys. Rev. Lett. 81, 1562 (1998).

[2] Super-Kamiokande Collab., S. Fukuda et al., Phys. Rev. Lett. 85, 3999 (2000).

[3] J.N. Bahcall, P.I. Krastev and A.Yu. Smirnov, Phys. Rev. D60, 093001 (1999); M.C. Gonzalez-Garcia, P.C. de Holanda, C. Pena-Garay, J.W.F. Valle, Nucl. Phys. B573, 3 (2000); G.L. Fogli, E. Lisi, D. Montanino, A. Palazzo, Phys. Rev. D62, 013002 (2000).

[4] M. Gell-Mann, P. Ramond and R. Slansky, in Supergravity, Proceedings of the workshop, Stony Brook, New York, 1979, edited by P. van Nieuwnehuizen and D. Freedman (NorthHolland, Amsterdam, 1979), p.315; T. Yanagida, in Proceedings of the Workshop on the Unified Theories and Baryon Number in Universe, Tsukuba, Japan, 1979, edited by O. Sawada and A. Sugamoto (KEK Report No. 79-18), Tsukuba, 1979), p.95; R.N. Mohapatra and G. Senjanović, Phys. Rev. Lett. 44, 912 (1980).

[5] C.H. Albright, K.S. Babu and S.M. Barr, Nucl. Phys. Proc. Suppl 77, 308 (1999); G. Altarelli and F. Feruglio, Phys. Lett. B439, 112 (1998). Z. Berezhiani, Z. Tavartkiladze, Phys. Lett. B409, 220 (1997); Z. Berezhiani and A. Rossi, JHEP 9903, 002 (1999).

[6] K.S. Babu and S.M. Barr, Phys. Lett. B381, 202 (1996).

[7] Z. Berezhiani, A. Rossi, Phys. Lett. B367, 219 (1996); S. F. King, Nucl. Phys. B562 57, (1999).

[8] A.Yu. Smirnov, Phys. Rev. D48, 3264 (1993).

[9] K.S. Babu, C.N. Leung and J. Pantaleone, Phys. Lett. B319, 191 (1993); P.H. Chankowski and Z. Pluciennik, Phys. Lett. B316, 312 (1993); J. Ellis and S. Lola, Phys. Lett. B458, 310, (1999); K.R.S. Balaji, A.S. Dighe, R.N. Mohapatra and M.K. Parida, Phys. Rev. Lett. 84, 5034 (2000); Phys. Lett. B481, 33 (2000); P.H. Chankowski, A. Ioannisian, S. Pokorski, J.W.F Valle, hep-ph/0011150 and references therein.

[10] A. Zee, Phys. Lett. B93, 389 (1980); K.S. Babu, Phys. Lett. B203, 132 (1988).

[11] S.M. Barr and I. Dorsner, Nucl. Phys. B585, 79 (2000); Z. Berezhiani and A. Rossi, Nucl. Phys. B594, 113 (2001); H. Fritzsch and Z.Z. Xing, hep-ph/9912358 and references therein; G. Altarelli, F. Feruglio, Phys.Rept. 320 295, (1999).

[12] D.O. Caldwell and R.N. Mohapatra, Phys. Rev. D 48, 3259 (1993); J. Peltoniemi, J.W.F. Valle, Nucl. Phys. B406, 409 (1993).

[13] S. Goswami, Phys. Rev. D 55, 2931 (1997).

[14] K.S. Babu and Q. Shafi, Phys. Lett. B311, 172 (1993); Y. Achiman and T. Greiner, Phys. Lett. B329, 33 (1994); K. Matsuda, T. Fukuyama and H. Nishiura, hep$\mathrm{ph} / 9906433$.

[15] T. K. Kuo, Guo-Hong Wu, Shao-Hsuan Chiu, Phys. Rev. D62 051301, (2000); hep$\mathrm{ph} / 0011058$.

[16] J. Peltoniemi, hep-ph/9506228.

[17] A. Kusenko, G. Segre, Phys. Lett. B369, 197 (1997); A. Kusenko, astro-ph/9903167.

[18] E. Nardi and J. I. Zuluaga, astro-ph/0006285.

[19] W. Kluzniak, astro-ph/9807224.

[20] G. F. Giudice, E. W. Kolb and A. Riotto, hep-ph/0005123.

[21] B. de Carlos, J.A. Casas, F. Quevedo, E. Roulet, Phys. Lett. B318:447, (1993).

[22] O.L.G. Peres and A.Yu. Smirnov, hep-ph/0011054. 
[23] CHOOZ Collab., M. Appolonio et al., Phys. Lett. B420, 397 (1998).

[24] LSND Collab., G. Mills et al., talk at Neutrino 2000.

[25] Bugey Collab., B. Achkar et al., Nucl. Phys. B434, 503 (1995).

[26] CDHS Collab., F. Dydak et al., Phys. Lett. B134, 281 (1984).

[27] E.J. Chun, Anjan S. Joshipura and A.Yu. Smirnov, Phys. Lett. B357, 608, (1995).

[28] M. Cvetić and P. Langacker, Phys. Rev. D54, 3570 (1990).

[29] K. Benakli and A. Yu. Smirnov, Phys. Rev. Lett 79, 4314 (1997); Gia Dvali and Y. Nir, JHEP 9810:014, (1998); N. Arkani-Hamed, L. Hall, H. Murayama, D. Smith and N. Weiner, hep-ph/0007001, hep-ph/0006312. 